\title{
How social-media and web-accessible learning resources influence students' experiences in a quantum physics course: A case study
}

\author{
Brandon James Johnson, Erin Ronayne Sohr, and Ayush Gupta \\ Department of Physics, University of Maryland, College Park, MD 20742
}

\begin{abstract}
As part of a project studying student experiences in quantum mechanics (QM), we collected two streams of data. We asked students to generate autobiographical video, audio, and written blogs and participate in openformat interviews while taking the first semester of an upper-division QM course. We also interviewed former students about their experiences in QM. In asking students to generate data on their own, we intended for students to have the freedom to generate their own critiques and appraisals of the class content and culture, including how it interacts with their experiences learning physics in other classes and their experiences outside of physics. While exploring preliminary themes, we present one student's experiences as informed by her use of "outside resources" (online solutions and examples, a class group-chat, and out-of-class study groups). We discuss research and instructional implications, specifically, how interactions with outside resources may be gendered and affect her emotions, moral stances, epistemology, and physics self-efficacy.
\end{abstract}

\section{INTRODUCTION}

As technology has progressed, so has access to online resources and social media platforms. Some of these resources used by students are outside of the expectations outlined by instructors in their courses. For the purposes of this paper, we call these "outside resources," which would include, for example, online solutions and examples, class group-chats and Facebook groups, online video lectures on platforms like YouTube and Khan Academy, etc., so long as these were not specifically elicited by the instructor. Access to new outside resources has likely changed the ways students approach learning. Due to the recency of these changes, the literature around how outside resources affect education is still fairly limited.

The use of outside resources, when not explicitly sanctioned, could be deemed illegitimate and framed as cheating [1]. However, when students use resources that are un-sanctioned, they do not always intend to cheat (for example, students may misunderstand what counts as plagiarism in a specific situation [2]). There are also significant differences between faculty and students' understanding of academic integrity and use of resources outside of class materials [3]. Finally, students' behaviors are influenced strongly by their perceptions of their peers' beliefs about cheating [3,4].

Like [4], our work privileges student intentions. In contrast to the previous literature, our starting orientation is to trust that even when students are using outside resources, they may not be motivated by a desire to cheat or break rules. Our work approaches use of outside resources qualitatively which privileges personal accounts and experiences. As such, it expands on the knowledge of how and why students use outside resources and how this use intersects with the many aspects of students' experiences both within and outside their coursework.

\section{METHODS}

We collected two sets of data as part of a project studying student experiences in upper-division quantum mechanics (QM). This research was motivated by positing students as experts of their own learning through explicit consideration of their experiences in their courses. The authors were also motivated by ethical considerations around whom this data and research serves, toward creating an equitable stance for all stakeholders in the learning process.

Our intention for this research was to record rich, authentic representations of students' experiences while learning QM. To this end, we intended for students to have the freedom to generate their own critiques and appraisals of the class content and culture, including how it interacts with their experiences learning physics in other classes and their experiences outside of physics.

Hence for the first dataset, we asked students enrolled in the first semester of an upper division QM sequence (current students) to generate autobiographical video, audio, and written blogs and participate in semi-structured interviews [5]. We asked them to submit short blogs once or twice a week and told them about our interest in their experience learning QM. Beyond these loose guidelines, students chose the format and content of their submissions. The students own the data, so they can use it for their own purposes if desired. The interviews were intended to be conversational and formatted specific to each participant based on their submissions and preliminary cross-cutting themes. 6 students volunteered to submit autobiographical submissions and be interviewed 2 or 3 times during the semester.

For the second dataset, we asked students who had previously taken the aforementioned course (past students) to participate in more structured interviews. These interviews were designed to have students reflect on their experience in the class, both the content and the culture. 11 students participated in these one-time, hour-long interviews. 
After the first few submissions from the current students and interviews with the first 4 past students, the authors noticed some preliminary themes. One theme we noticed was that several students talked about using the solutions manual available on the internet. Additionally, some students talked about use of online video lecture platforms and of group textbased communication platforms. We considered these to be thematically connected and termed them all "use of outside resources." The manner of use of these resources varied from student to student. Students (even those with the same instructor) relayed different interpretations of their instructor's expectations with regard to outside resources.

To start analysis, sections of 4 interviews with past students and submissions and interviews from 4 current students that contained references to outside resources as remembered by the researchers were transcribed and collated. These sections were brought to research meetings, where the research team (authors and Andrew Elby) discussed the data [6]. Preliminary analysis at this stage explored interpreting the data using constructs of epistemology [7], self-efficacy [8], ethical reasoning [3], identity [9], etc. The modeling of the data explicitly using these constructs is left for future detailed analysis. However, we mention this here because the preliminary analysis below follows from these discussions, and we do draw on these constructs for the preliminary interpretations we present in this paper. In this paper, we focus on one student in our dataset, Shawn (pseudonym). Shawn addressed the challenges and affordances of outside resources at length, especially how they informed her experience in the class. Because of this and space restraints of a conference paper, we decided to focus on Shawn's submissions and interviews. In the sections below, we first present segments from the interviews with Shawn, limiting ourselves to low-inference claims. Subsequently, we present our preliminary analysis that includes higher level inferences based on the data.

\section{INTERVIEW CONTEXT AND DATA}

At the time of the study, Shawn was in the second semester of her third year as a physics and astronomy double major. She was enrolled in a full-time load of courses including the first semester of the upper division QM sequence. She was also involved in a research group and several student organizations. Outside of school, she lived at home and often used her time to deepen her relationships with family and long-time friends. She also worked as a nanny part-time and taught religious education at her church on Sundays.

For this study, the first author, Brandon, interviewed Shawn approximately one month after the start of the semester. At that time, Shawn had submitted 8 autobiographical artifacts as a part of this research project. The content of her submissions was varied. She reflected on difficulties during the first homework assignment, anxiety about preparing for her first QM test, the life-intersections between school and home, current events in the world, and her decision to quit her nannying job to focus on school.

Before the interview, Brandon created interview prompts based on Shawn's submissions as well as preliminary crosscutting themes from the larger data set. These prompts included, for example, asking about online solutions, time management, and going to office hours. Brandon's intention for the interview and prompts was to create a setting which allowed for casual discussion where the conversation can follow topics that both participants bring up. During the interview, Shawn was quite self-generative, often talking for several minutes with only gestural or affirmative vocal responses, like nods, 'mmhmm's, and 'sure's, from Brandon.

The authors chose a 15-minute segment toward the end of this interview for further analysis because of its length and depth with regard to outside resources. The following is a summary of this section of the interview where, due to space constraints, we are quoting only passages which are needed to maintain the story as portrayed by Shawn.

This section of the interview starts when Brandon asks Shawn about how she identifies with respect to gender, race, and sexual orientation. In response, Shawn relays several experiences where her gender affected her experience in the physics department. Shawn starts by explaining that she has "not genuinely experienced [sexism] from anyone who is a higher authority figure than [her]." She goes on to describe how she "feel[s] very valued" in her research group. However, she contrasts this by describing interactions between her and some of her male peers in a course context.

"It's never like, 'Oh you're dumb 'cause you're a girl,' kind of stuff. But it's when you go to study groups, and [...] you say like, 'Oh maybe we can solve it this way.' And they question you for two hours about why you think that's a good idea and 'Where are your sources?' and what page in the textbook it came from and things like that. Versus, the guy says the same thing and, 'Oh okay, that sounds good,' to the point that all the girls in my cohort do not study with specific men anymore, and we have our own study group because we could not handle it. It was so bad. It was just, it was horrible."

Continuing, Shawn also describes some interactions via a whole-class group-chat.

"I would ask a question and, people, other guys would just kind of respond with, 'Well if you did it yourself, you would understand it.' And I'm sitting here like, 'You don't know anything about me as a person.' [...] I have clearly not enough time or had had not enough time to really in-depth read the book, and I'm trying to get more conceptual information of like, 'Okay I think this matches with this problem. Can you tell me if this equation goes with this problem before I like spend forty minutes doing the wrong thing?' And it would just be a lot of like you know, 'Well if you paid attention, you would understand it.' And I feel like as much as that hurts, it wouldn't have bothered me as much 
as if, if it hadn't happened at least like four times now where an hour later, a different person who was a guy would ask the same question and be met with, 'Oh okay here, let me help.' And I'm just like ((puts hands on cheeks)), 'Ah! What is happening!?",

At this point in the interview, Shawn talks for about 5 minutes about some interactions she has had in one of the student organizations she is involved with which she describes as "agrivat[ing]." Afterwards, Brandon redirects the conversation towards an aspect of Shawn's first submission where she searched for solutions online. When Brandon asked Shawn about her feelings toward looking up online solutions, she answers, "It's like a test of endurance. How long do I want to beat my head against the wall to try to figure it out on my own? "She then references back to the class group-chat describing how she responds to the type of responses she receives when asking for homework help:

"I get like a lot of responses like, 'You should know this already.' [...] And that becomes this whole internalized thing of me trying to really hard and spending like two hours on one problem on like trying to figure it out."

Then she explains what happens if she eventually looks things up online.

"Sometimes I can find something online where it's similar enough but not the exact problem that I'm like, 'Okay, I still have to take the time to like actually apply this method to this problem.' But when I find like the entire solution, I'm like, almost always I look at it, I'm like, 'Dude, I completely understand what's going on, 'but the fact that I can't seem to make that connection myself half the time really bothers me. And it feels like, like you said, like I'm almost cheating or I'm caving."

Perhaps as justification for actions which Shawn considers to be cheating, she emphasizes her values by comparing herself to some of her peers, especially those whom Shawn portrays as "spend[ing] like twelve hours" on homework. She states:

"I like being a multifaceted person. I like going to my church and teaching on Sundays. I like the fact that I've been working, and I have things to do. [...] You know, [these peers are] not even involved in research. [They're] just like, '[...] I do like two classes, and I spend like literally all my time focusing on like two classes.' And I'm like, 'I'm glad you can. I can't.' [...] It feels like I'm cheating, or not trying as hard as people feel like I should. And I try as hard as I can. [...] Maybe it's just me not being as smart as I should be as a physics major, but I need to do these extra steps."

Shawn explains that studying in a group can alleviate some of these feelings: "If I'm studying in a group and everyone seems confused about the same thing, that helps because it's like, I'm not just like the only one who's so dumb and can't do this. "However due to her commute from home and part-time nannying job, she is often unavailable for evening study groups.

"It sucks to feel like I'm asking all these questions, and I get horribly embarrassed even asking sometimes in the allgirls study group-chat [...] when it gets to like the sixth question on the same problem because I can't remember how to do algebra, I'm just like (sigh). Okay, I'm gonna go look it up."

Shawn continues by describing that she often gets stuck while attempting to recall certain math techniques.

"I really want to try really hard to be able to just feel comfortable enough with a problem to just look at it and be like, 'Obviously this is exactly what I know how to do.' [...] And it's always, it's always like the math of it, you know. Where I'm sitting here, I'm like 'I know this is the integral I need to do, but how?' And that's when I have to cave and look it up. And it's just kind of, like, because it's stuff like that. When it's usually like, 'Oh I messed up on the algebra,' or, 'I forgot how to u sub in an integral,' and stuff like that. I'm just like, 'You should know this by now Shawn.",

\section{PRELIMINARY ANALYSIS}

In this section, we retell Shawn's story interpreted through researchers' perspectives, and model preliminary or tentative connections to epistemology, ethical stances, identity, and self-efficacy as appropriate.

In the above conversation, Shawn states that she makes use of several outside resources when doing homework in her QM course. These resources include searching the internet for solutions and examples, asking questions through an all-class and all-girls group-chats, and participating in study groups. Here we reflect on some of the themes in the conversation that were salient to Shawn and, upon reflection, to us.

The first theme we highlight is how, according to Shawn, gender (hers' and her peers') seemed to shape her interactions in study groups and the group-chat. In her storytelling, Shawn connects the kind of marginalization she faces in these spaces to her gender. To highlight this, she contrasts how her peers respond positively to other male peers when they ask questions or propose ideas. Due to the gender-based hostility of this environment, Shawn and her other female classmates take measures to avoid working with some of their peers.

However, interactions with her peers affect how Shawn approaches homework and learning in the course. She describes homework as a "test of endurance," feeling pressure to spend a lot of time trying to solve the problems without help from online resources or peer-groups. And in some ways, she has associated being able to do the homework problems on her own as evidence of learning, even when she feels that ultimately, she would have to "cave" and look up the information. 
This also has consequences for how Shawn thinks about the morality of using outside resources. Several times she mentions how she feels that looking up resources to support her in answering homework counts as "cheating." However, at other times she acknowledges the various demands on her time that she feels many of her peers do not have. In this way, there appears to be some nuance to Shawn's ethical stance regarding outside resources. Another nuance Shawn brings to the notion of cheating is distinguishing between looking up the exact solution to a problem, versus looking up solutions to similar problems.

All of this has consequences for Shawn's confidence in her own ability to learn and do physics (self-efficacy). During the interview, she shows uncertainty in her abilities compared to what she thinks a physics student should be able to do. "Maybe it's just me not being as smart as I should be as a physics major." "You should know this by now Shawn." Her statements mimic the types of comments she received from her peers. Similarly, when she says, "How long do I want to beat my head against the wall to try to figure it out on my own?" she has already framed the homework activity in a way that admits "defeat" which for Shawn is "caving" and looking things up.

In summary, we argue that Shawn's use of outside resources is, in part, entangled with her interactions with her peers on the course-related group-chat, specifically the ways in which she feels she is marginalized because of her gender. Stemming from these interactions, the use of outside resources is linked to Shawn's emotions, her view of learning and doing physics in the course (epistemology), her moral stances, and to her self-efficacy with respect to physics.

\section{IMPLICATIONS AND CONCLUSIONS}

While we cannot generalize to the whole student population, Shawn's case serves as a revelatory case, that helps shed light on some of the nuances that might need consideration in discussions of outside resources [10]. In that sense, it provides theoretical generalizations, rather than statistical generalization [11]. What we learn from looking at Shawn's case, is that students interact with outside resources in ways that instructors might not focus on or know about. For example, some students may have nuanced

[1] D. L. King and C. J. Case. Issues in Information Systems 15, 1 (2014).

[2] S. Yeo. High Ed. Research \& Development 26, 2 (2007) p. 199-216.

[3] D. D. Carpenter et al. Journal of Engineering Ed. 95, 3 (2006) p. 181-194.

[4] J. M. Stephens, M. F. Young, and T. Calabrese, Ethics \& Behavior 17, 3 (2007) p. 233-254.

[5] S. E. Rabionet. Qualitative Report 16, 2 (2011) p. 563 566.

[6] A. H. Schoenfeld. The Journal of the Learning Sciences 2, 2 (1992) p. 179-214. differentiations between copying the exact solution, using a similar problem, referencing a textbook, or asking peers in person or via social media. These interactions have consequences for learning. As in [4], our data shows how for students like Shawn, their perceived expectations of peers are entangled with their behaviors. We also saw how interactions in online spaces can marginalize students like Shawn. These interactions may also have consequences for students' self-efficacy with respect to the course and the discipline, which, in turn, can impact their learning [12]. The extent to which Shawn considers ethical motivations in approaching homework contrasts those studies that tend to frame students as morally or epistemologically naïve [1].

We feel that instructors should be cognizant of the increasing number of outside resources and have explicit conversations with students about the responsible use of outside resource use towards supporting their learning. Given Shawn's experience in the group-chat, we feel that it is also important for instructors to discuss norms for socialmedia related communications so that students are more mindful of avoiding comments that can marginalize and alienate others. Classroom conversations on implicit bias and micro-aggression could help students be reflective about their own interactions as well as develop skills for acting as a responsible bystander towards resisting marginalizing behaviors. Additionally, instructors cannot control all their students' actions but can make themselves available to talk about outside resource use and be a support for students who might be facing marginalization.

We need a better understanding of students' views on outside resource use. Research communities should strive to better understand how learning happens with use of outside resources, including how it interacts with their overall experiences inside and outside of class.

\section{ACKNOWLEDGEMENTS}

We thank Andrew Elby, Hannah Sabo, and the PER group at UMD, and Kathleen McPartland for ideas and suggestions. This work was supported by NSF DUE \#1625797.
[7] A. Elby, C. Macrander, \& D. Hammer. Handbook of epistemic cognition, (2016) p. 113-127.

[8] V. Sawtelle, E. Brewe, \& L. H. Kramer. Journal of research in science teaching 49, 9 (2012) p. 1096-1121.

[9] M. Ong. Social problems 52, 4 (2005) p. 593-617.

[10]R. K. Yin. (2017). Case study research and applications: Design and methods. Sage publications.

[11] M. Eisenhart. (2009). In Generalizing from educational research, p. 61-76. Routledge.

[12] J. E. Dowd, I. Araujo, and E. Mazur, Phys. Rev. ST Phys. Educ. Res. 11, 1 (2015). 